\title{
Estudio cuantitativo de los factores que afectan el desempeño de los parques científico-tecnológicos (PCT)
}

\author{
Quantitative Study of the Factors that Affect \\ the Performance of Scientific-Technological \\ Parks (STP)
}

\section{Estudo quantitativo dos fatores que afetam o desempenho dos parques científico- tecnológicos (PCT)}

\author{
Gerardo Luís Angulo Cuentas* \\ Maryuris Charris Polo ${ }^{* *}$ \\ Jaime Camacho Pico ${ }^{* * *}$
}

Fecha de recibido: 1 de noviembre de 2013

Fecha de aprobado: 13 de mayo de 2014

Doi: dx.doi.org/10.12804/rev.univ.empresa.27.2014.08

Para citar este artículo: Angulo C., G. L., Charris P., M., \& Camacho P., J. (2014). Estudio cuantitativo de los factores que afectan el desempeño de los parques científico-tecnológicos (PCT). Universidad \& Empresa, 16(27), 23 1-257. doi: dx.doi.org/10.12804/rev.univ.empresa.27.2014.08

* Estudiante del Doctorado en Gestión y desarrollo tecnológico de la Universidad Industrial de Santander (UIS) (Colombia). Magister en Ingeniería Industrial de la Universidad del Norte (Colombia). Ingeniero Industrial de la Universidad del Atlántico (Colombia). Profesor Asociado de la Universidad del Magdalena (Colombia). Correo electrónico: gerardoangulo@unimagdalena.edu.co

** Magister en Ingeniería Industrial de la Universidad Industrial de Santander (UIS) (Colombia). Ingeniero Industrial de la Universidad del Magdalena (Colombia). Contratista del Despacho del Rector de la Universidad del Magdalena (Colombia).Correo electrónico: mcharris@unimagdalena.edu.co

*** Doctor en Ingeniería Industrial de la Universidad Politécnica de Cataluña (España). Ingeniero Industrial, Universidad Industrial de Santander (UIS) (Colombia). Profesor Asociado de la Universidad Industrial de Santander (UIS) (Colombia). Correo electrónico: jcamacho@uis.edu.co 


\section{RESUMEN}

Esta investigación pone a prueba, empíricamente, una serie de hipótesis sobre una muestra de 409 parques científico-tecnológicos (PCT) de todo el mundo. Esto se hace, mediante varios modelos de regresión, con el objetivo de analizar el impacto de factores internos y externalidades sobre el desempeño de los PCT. Los resultados más importantes muestran los casos y las condiciones en los que factores internos tienen un impacto positivo sobre el desempeño. Dada la escasa evidencia disponible, analizando los aspectos de los que trata este trabajo, el estudio aporta evidencia empírica significativa al avance de la literatura relativa al tema.

Palabras clave: administración de parques científico-tecnológicos (PCT), desempeño económico, desempeño innovador, parques científico-tecnológicos (PCT).

\section{ABSTRACT}

This research empirically puts to the test a series of hypotheses regarding a sample of 407 scientific-technological parks (STP) around the world. This is done using various regression models in order to analyze the impact of internalities and externalities on the performance of the STP. The most important results show the cases and conditions in which internal factors have a positive impact on performance. Given the scarce evidence available and by analyzing the aspects on which this study focuses, the work contributes significant empirical evidence to the advance of the literature on the subject.

Keywords: Economic performance, innovative performance, management of scientifictechnological parks (STP), scientific-technological parks (STP).

\section{RESUMO}

Esta pesquisa põe a prova, empiricamente, uma série de hipóteses sobre uma amostra de 409 parques científico-tecnológicos (PCT) do mundo todo. Isto se faz, mediante vários modelos de regressão, com o objetivo de analisar o impacto de fatores internos e externalidades sobre o desempenho dos PCT; os resultados mais importantes mostram os casos e as condições nos que fatores internos têm um impacto positivo sobre o desempenho. Dada a escassa evidência disponível, analisando os aspectos dos que trata este trabalho, o estudo aporta evidência empírica significativa ao avance da literatura relativa ao tema.

Palavras-chave: administração de parques científico-tecnológicos (PCT), desempenho económico, desempenho inovador, parques científico-tecnológicos (PCT). 


\section{INTRODUCCIÓN}

No existe una definición universalmente aceptada sobre los parques científico-tecnológicos (PCT)(Hansson, Husted, \& Vestergaard, 2005). De acuerdo con la International Association of Science Parks (IASP, 2002), un PCT es una "organización gestionada por profesionales especializados, cuyo principal objetivo es incrementar la riqueza de su comunidad promoviendo la cultura de la innovación y la competitividad de los negocios y de las instituciones basadas en conocimiento asociadas a él" (Sección Science park [IASP official definition], párr. 1). Entre otras actividades, este 1) estimula y gestiona el flujo de conocimiento y tecnología entre universidades, instituciones de investigación, empresas y mercados, 2) impulsa la creación y el crecimiento de empresas innovadoras mediante mecanismos de incubación y de generación centrífuga (spin-off) y 3) proporciona otros servicios de valor agregado así como espacio e instalaciones.

Para la Asociación de Parques Científicos y Tecnológicos de España (APTE, 2003) un PCT es un proyecto, generalmente asociado a un espacio físico, que 1) mantiene relaciones formales y operativas con las universidades, centros de investiga- ción y otras instituciones de educación superior, 2) está diseñado para alentar la formación y el crecimiento de empresas basadas en el conocimiento y de otras organizaciones de alto valor agregado pertenecientes al sector terciario, normalmente residentes en el propio parque, y 3) posee un organismo estable de gestión que impulsa la transferencia de tecnología y fomenta la innovación entre las empresas y organizaciones usuarias del parque.

De acuerdo con la United Kingdom Science Park Association(UKSPA, 2011), un PCT es esencialmente un aglomerado de negocios basado en el conocimiento, en donde se suministra apoyo y asesoría para contribuir al crecimiento de las empresas. En la mayoría de los casos, los parques científicos están asociados con un centro de tecnología como una universidad o un instituto de investigación. Un PCT típico ofrece servicios de propiedad, tecnología y negocios. Estos pueden ser 'en casa' (in house) o a través de una red de expertos. En los PCT cuatro características se destacan: 1) la flexibilidad de los términos del arrendamiento, 2) el apoyo en tecnología a través de una universidad u organización asociada de investigación, 3) la existencia de servicios básicos de negocios (recepción, telecomunicaciones de banda ancha, fotocopiado, 
limpieza, seguridad, servicio de secretariado y de alquiler de salas de conferencias) que le permiten a una empresa operar desde el primer día y 4) servicios avanzados (transferencia de tecnología, asesoramiento en propiedad intelectual, acceso a préstamos y capital de riesgo, prácticas de estudiantes, consejos de marketing, etc.).

La Association of University Research Parks (AURP, 2011) define un PCT como una iniciativa basada en la propiedad, que 1) desarrolla planes maestros de propiedad diseñados para la investigación y comercialización, 2) crea alianzas con universidades e instituciones de investigación, 3) estimula el crecimiento de nuevas empresas, 4) transfiere tecnología y 5) conduce al desarrollo económico basado en tecnología.

Finalmente, para Castells y Hall (1994) un PCT es un área empresarial de alta tecnología deliberadamente establecida como resultado de iniciativas gubernamentales o provenientes de las universidades. Según los autores, este aspira a inducir un nuevo crecimiento industrial, en términos de empleo y producción, intentando atraer empresas de producción de alta tecnología hacia un espacio privilegiado.

Como se observa en las múltiples definiciones revisadas, existen al menos tres elementos indispensables para la conformación de un PCT. Estos son: 1) la existencia de espacios físicos disponibles, en venta o en arriendo, para el establecimiento de empresas, 2) la presencia de universidades $u$ otros centros de innovación y producción de conocimiento o la constatación de vínculos estrechos con estos y 3) una administración que gestiona el flujo de conocimientos y tecnología, fomenta la creación y el crecimiento de empresas innovadoras y proporciona servicios de valor añadido, entre otras funciones. Esto con el objetivo de propiciar desarrollo económico basado en ciencia, tecnología e innovación en el territorio de influencia del PCT.

Considerando lo anterior, es decir, los objetivos, metas, retos y medios de actuación de los PCT, resulta de interés, tanto para los gobiernos y organizaciones que pretenden implementarlos, como para los gestores de PCT, estudiar los factores internos y externos que afectan su desempeño. Este es, precisamente, el objetivo del presente trabajo.

\section{MARCO TEÓRICO}

\section{A. El desempeño de los PCT}

En los últimos años, se ha estudiado el desempeño de los PCT en di- 
versos países. En Suecia, Dettwiler, Lindelöf y Löfsten (2006), Ferguson y Olofsson (2004), Lindelöf y Löfsten $(2002,2003,2004)$ y Löfsten y Lindelöf (2002, 2003, 2005); en Taiwan, Chen, Wu y Lin (2006), Sun (2011) y Yang, Motohashi y Chen (2009); en el Reino Unido, Hansson et al. (2005), Siegel, Westhead y Wright (2003a, 2003b) y UKSPA (2003); en China, Hu (2007) y Tan (2006); en Italia, Bigliardi, Dormio, Nosella y Petroni (2006) y Colombo y Delmastro (2002); en Dinamarca, Hansson et al. (2005); en Finlandia, Squicciarini (2009); en Japón, Fukugawa (2006); en los Estados Unidos, Link y Link (2003) y Link y Scott (2003), y en Grecia, Bakouros, Mardas y Varsakelis (2002). Evaluar el desempeño de los PCT puede ser una tarea compleja, para la que es posible hacer uso de múltiples enfoques que, lamentablemente, no son infalibles (Bigliardi et al., 2006).

El enfoque predominante para evaluar el desempeño ha sido la estimación del valor agregado por los PCT a las firmas hospedadas. Esto mediante el estudio de bases de datos o la aplicación de encuestas para recolectar, analizar y contrastar información de las empresas que están dentro o fuera de ellos. Lo anterior, se hace con el fin de verificar diferencias que confirmen la existencia de dicho valor agregado. Las métricas utilizadas para estimar este valor son de tres tipos: 1) de desempeño financiero (p. ej., crecimiento en ventas o ingresos), 2) de desempeño innovador (p. ej., número de patentes, derechos de autor y creación de nuevos productos) y 3) de desempeño en materia de generación de empresas (p. ej., compañías incubadas, supervivencia de las mismas y crecimiento en empleo).

Link y Link (2003) encuentran que los directores de los PCT en Estados Unidos miden el desempeño de su parque de una variedad de maneras, incluyendo en estas a la rentabilidad, las contribuciones a la economía local y regional y la capacidad de interactuar con las universidades.

A pesar de la diversidad de medidas que los directores mencionan, todas están atadas de una u otra forma en la habilidad del parque para crecer en términos de empresas o empleados. Link y Scott (2003) argumentan que el Research Triangle Park es el más notable y exitoso de todos los parques científicos en Estados Unidos, con un crecimiento sostenido, en términos de empresas y empleos de investigación, desde finales de la década del cincuenta. Ferguson y Olofsson (2004) estudian firmas dentro y fuera de los PCT en Suecia y encuentran que, 
durante el período 1995-2002, las empresas localizadas en estos espacios tuvieron una mejor tasa de supervivencia que aquellas ubicadas por fuera de los mismos.

En cuanto al crecimiento en el empleo y las ventas, durante el período existente entre 1991 y 2000 , se encuentra que, si bien las firmas dentro de PCT presentan promedios y medianas superiores que las que se encuentran afuera, la alta desviación estándar en ambas métricas hace concluir que no existen diferencias estadísticamente significativas entre las empresas de ambos grupos. Estos resultados son consistentes con lo afirmado por Siegel (2003) quien, al revisar la literatura, encuentra que Westhead y Storey (1994) y Westhead (1997) calculan las diferencias en los valores medios de los indicadores de desempeño para varias firmas dentro y fuera de PCT, y no encuentran diferencias estadísticamente significativas en la probabilidad de supervivencia y la creación de empleo.

Löfsten y Lindelöf (2005) analizan 134 firmas ubicadas en PCT para comparar el rendimiento de spinoffs universitarios frente a spin-offs corporativos, utilizando como métricas el crecimiento en ventas y la rentabilidad en ambos grupos. Estos autores no encuentran diferencias estadísticamente significativas. Sun (2011), por su parte, intenta medir el desempeño de seis industrias de alta tecnología en el Hsin Chu Industrial Science Park en Taiwán para el periodo 2000-2006. Para esto utiliza las que considera dos bien conocidas medidas del desempeño total: el número de patentes y las ventas anuales.

Siegel et al. (2003a) afirman que Westhead (1997) y Westhead y Storey (1994), para valorar el impacto de los PCT en materia de productividad científica, calculan diferencias en los valores medios de indicadores de desempeño tales como inversión en $\mathrm{I}+\mathrm{D}$, número de científicos e ingenieros, número de patentes y derechos de autor y creación de nuevos productos en varias firmas dentro y fuera de los PCT. Estos autores no encuentran tampoco diferencias estadísticamente significativas.

Para determinar si las firmas ubicadas en el Hsinchu Science and Industrial Park (HSIP) son más innovadoras que las ubicadas afuera, Yang et al. (2009) comparan los siguientes indicadores: inversión en I+D (en millones de nuevos dólares taiwaneses NT\$, tanto para el nivel del PCT como el de Taiwán), intensidad de la $\mathrm{I}+\mathrm{D}$ (inversión en $\mathrm{I}+\mathrm{D} /$ PIB para el nivel Taiwán, inversión en I+D/ventas, para el nivel del PCT) 
y las patentes de invención (número de estas para ambos niveles). Se encuentra que el promedio en $\mathrm{I}+\mathrm{D}$ de las empresas del HSIP es 5,37\%, es decir, casi 2,77 veces la media I+D para todas las empresas en la muestra periodo. En cuanto a I+D y las patentes de invención concedidas, observaron que el porcentaje que corresponde a empresas HSIP aumentó de $4,79 \%$ en 1990 a $20,93 \%$ en 2003 , en relación con la $\mathrm{I}+\mathrm{D}$, y del $14,04 \%$ al $35,46 \%$, en lo relativo a las patentes de invención. Este aumento puede ser en parte atribuido al número creciente de empresas en el HSIP (cerca de 400 empresas se encontraban en este parque en 2003).

Estas estadísticas revelan, entonces, que el rendimiento de las empresas HSIP es generalmente mejor que aquel de las que están por fuera del parque, tanto en términos de $\mathrm{I}+\mathrm{D}$ como de aplicación exitosa de patentes. Sun (2011) que, como ya se ha anunciado, intenta medir el desempeño de seis industrias de alta tecnología en el HSIP, utiliza para esto, como medidas del desempeño total, el número de patentes y las ventas anuales.

Como puede observarse, es posible utilizar estos indicadores para comparar tanto el desempeño de las firmas ubicadas en los PCT, como aquel del parque como unidad de análisis.
Considerando la disponibilidad de datos que ofrece el Atlas Mundial de la Innovación (The World Atlas of Innovation de la World Alliance for Innovation [WAINOVA], 2009), se han seleccionado como mediciones de desempeño financiero, innovador y en generación de empresas (las resumidas en la tabla 2). Estas se detallan más adelante.

B. Factores asociados al desempeño de PCT

A partir de la revisión de la literatura relacionada con el desempeño de los PCT y de la disponibilidad de datos empíricos, se decidió explorar el efecto de los siguientes factores: diversidad de sectores tecnológicos, infraestructura física, edad del parque, presencia de centros de $\mathrm{I}+\mathrm{D}$ y esfuerzo en incubación de empresas. Como variables de control y, pretendiendo descartar su posible relación con alguna medida del desempeño del PCT, se han considerado las siguientes magnitudes del país donde se ubica cada parque: solicitudes de patentes, población, PIB real y fuerza laboral.

Diversidad de sectores tecnológicos

Link y Link (2003) compara parques universitarios con criterios sobre inquilinos (PUCI) contra los parques 
universitarios sin criterios sobre inquilinos (PUSCI). Concluyen que los primeros tienden a estar más cerca de una universidad de investigación que los segundos y que los criterios sobre admisión de inquilinos parecen ser determinantes en términos de atraer más rápido empresas y, por lo tanto, más empleados. Para el gobierno de Taiwán, en donde el espacio en los PCT es muy limitado, la selección de las empresas se ha convertido en un tema crítico. Se opta por aquellas que tienen un mayor potencial de crecimiento y pertenecen a determinados sectores de alta tecnología (Chen et al., 2006). Los sectores industriales son escogidos cuidadosamente.

En concreto, para poder ser aceptado en el HSIP, un sector debe cumplir por lo menos uno de los siguientes criterios: 1) contar con diversas capacidades en desarrollo y fabricación de productos y con un plan integral para el desarrollo de los mismos, 2) sus productos deben tener potencial para el desarrollo y la innovación, 3) ser intensivo en $\mathrm{I}+\mathrm{D}$ o ayudar a introducir o formar científicos avanzados y técnicos durante el proceso de fabricación y 4) estar representado por un instituto de investigación bien establecido que este centrado en $\mathrm{I}+\mathrm{D}+\mathrm{i}$ (Yang et al., 2009). Atendiendo a la literatura y a los datos disponibles se plantea entonces contrastar las siguientes hipótesis:

H1: la diversidad de sectores tecnológicos de las empresas ubicadas en un PCT está asociada con el desempeño del PCT.

\section{Infraestructura física}

Los PCT atraen a un número significativamente mayor de empresas de base tecnológica (EBT) que otras iniciativas. Los factores más importantes para atraer firmas a estos espacios son los atributos físicos, por encima de la infraestructura de servicios de apoyo (UKSPA, 2003). Dettwiler et al. (2006) plantean que la gestión de instalaciones en los PCT es un elemento fundamental que contribuye a la mejora del entorno empresarial y que es un factor explicativo del rendimiento y crecimiento de las compañías que se encuentran en estos. También argumentan que la gestión de la infraestructura en PCT contribuye a la generación de escenarios para la interacción, las relaciones entre empresas y la conformación de redes. Confirman sus planteamientos a través de una encuesta aplicada a 134 firmas ubicadas dentro y fuera de 10 PCT en Suecia.

Como se ha indicado, el espacio físico disponible, en venta o en arriendo, para el establecimiento de empresas es uno de los tres elementos indispen- 
sables para la conformación de un PCT(APTE, 2003;AURP, 2011; Castells y Hall, 1994; IASP, 2002; UKSPA, 2011). Atendiendo a la literatura y los datos disponibles se plantea contrastar la siguiente hipótesis:

H2: La infraestructura física está asociada con el desempeño del PCT.

La edad del PCT

En la literatura no fue posible encontrar estudios específicos acerca del efecto de la edad sobre el desempeño de los PCT. Sin embargo, Bigliardi et al. (2006) destacan que la misión y, en consecuencia, la "verdadera estrategia" de un parque, solo surge después de que este ha existido durante un período determinado de tiempo, en el cual se ha aprovechado de las oportunidades que se le han ofrecido, ha creado su propio 'espacio vital' y ha consolidado sus estructuras. Por esta razón, el modelo de negocio y la conducta estratégica vigentes en un PCT se ven influenciados por estrategias implícitas que no coinciden necesariamente con las misiones y los objetivos que se declararon formalmente en el momento de su creación. Atendiendo a la literatura y a los datos disponibles, se plantea contrastar la siguiente hipótesis:

H3: La edad del PCT está asociada con su desempeño.
La presencia de centros de I+D

La presencia de vínculos estrechos con universidades, centros de innovación y producción de conocimiento es uno de los tres elementos indispensables para la conformación de un PCT y para el logro de sus objetivos básicos (APTE, 2003; AURP, 2011; Castells y Hall, 1994; IASP, 2002; UKSPA, 2011). Löfsten y Lindelöf (2002) resaltan que las empresas ubicadas en PCT tienen un interés marcado en el acceso a los equipos, a los resultados de I+D y al personal calificado de los centros de I+D. Esto con el propósito de lanzar nuevos productos y servicios. Ferguson y Olofsson (2004) encuentran que las empresas ubicadas en estos lugares tienen tasas más altas de supervivencia.

Tanto la percepción de los empresarios, como los datos analizados asocian la cooperación con centros de I + D positivamente con el crecimiento de firmas en términos de empleo y ventas. Hanson et al. (2005) plantean que la función especial de los PCT ha sido proporcionar proximidad entre los investigadores que trabajan en diferentes instituciones y empresas, aumentando así la interacción y la transferencia de conocimientos científicos que son valiosos en contexto comercial. Sin embargo, 
reconocen que estos no han logrado desempeñar una función notable en el establecimiento de relaciones en red entre los investigadores universitarios y las empresas ubicadas en su interior.

Como puede observarse, existen resultados contradictorios, por lo que, atendiendo a los datos disponibles, valdría la pena contrastar la siguiente hipótesis:

H4: La presencia de centros de I+D está asociada con el desempeño del PCT.

\section{El esfuerzo en incubación de empresas}

Colombo y Delmastro (2002) afirman que los PCT son un elemento importante de una política de fomento a la creación de empresas de base tecnológica, especialmente en países con un sistema de innovación débil. En China, el Zhongguancun Science Park ha sido la fuerza detrás del crecimiento de las industrias intensivas mediante la atracción y consolidación del conglomerado más grande de empresas de alta tecnología en ese país (Tan, 2006).

Colombo y Delmastro (2002), al observar la considerable heterogeneidad de los PCT italianos y del Reino Unido, en cuanto a su organización y gestión, se preguntan si existe un modelo organizativo exitoso de PCT que surja de manera natural. La evidencia que encuentran sugiere que un factor de éxito incluye una organización interna esbelta y ágil y la coordinación efectiva de los servicios prestados por terceros, poniendo énfasis en la intermediación llevada a cabo por el personal del parque. Storey y Tether (1998) y luego Hansson et al. (2005) destacan los servicios de gestión prestados por el personal de los PCT dentro de las expectativas de emprendedores y pequeñas empresas de alta tecnología que pretenden ubicarse en estos.

Una función más activa propone Salvador (2011) para el personal de un PCT, al considerarlo como una efectiva fuerza de trabajo dedicada a abordar los problemas de financiación y el seguimiento constante de la mejora de las competencias de gestión, así como el logro de la credibilidad de las firmas hospedadas en el mercado. Así, atendiendo a la literatura y los datos disponibles se plantea contrastar la siguiente hipótesis:

H5: El esfuerzo en incubación de empresas está asociado con el desempeño del PCT.

\section{METODOLOGÍA}

En esta investigación se ponen a prueba, empíricamente, las hipótesis 
planteadas. Esto sobre una muestra de 409 PCT de todo el mundo (ver tabla 1). Este trabajo se hace mediante varios modelos de regresión, para analizar la relación de factores internos y externos con el desempeño. A continuación, se dan mayores detalles sobre la muestra, las variables y el tratamiento de los datos empleados con el fin de contrastar las mencionadas hipótesis.

Tabla 1. Distribución geográfica de la muestra analizada

\begin{tabular}{c|c|c}
\hline Continente & PCT & $\%$ \\
\hline África & 2 & $0,49 \%$ \\
\hline América & 118 & $28,85 \%$ \\
\hline Asia & 69 & $16,87 \%$ \\
\hline Europa & 213 & $52,08 \%$ \\
\hline Oceanía & 7 & $1,71 \%$ \\
\hline Total & 409 & $100 \%$ \\
\hline
\end{tabular}

Fuente: elaboración propia.

\section{A. Muestra}

Los datos del presente estudio empírico provienen de la información suministrada por la totalidad de los parques científicos tecnológicos presentes en el Atlas de Innovación (WAINOVA, 2009). Este recoge información acerca de más de 600 organizaciones basadas en tecnología (incubadoras de empresas, PCT y asociaciones nacionales e internacionales de los mismos) ubicadas en 76 países. Para el caso particular de los PCT, contiene información de 409 de estas organizaciones en 48 países.

\section{B. Variables}

En esta investigación se considera el desempeño de los PCT como variable dependiente. Se trabajaron tres formas diferentes para analizar el desempeño de estas organizaciones: el desempeño financiero, el desempeño innovador y el desempeño en generación de empresas. El primero es representado por los ingresos anuales totales en dólares estadounidenses recibidos por el PCT. El segundo es representado por dos mediciones, por un lado, las patentes comercializadas y, por el otro, las patentes solicitadas. El tercero se hace teniendo en cuenta tres mediciones: 1) las empresas graduadas desde la creación del PCT, 2) las incubadas y 3) las residentes. Todas las variables fueron observadas en 2007.

Para el análisis de los factores internos y externalidades que podían tener alguna relación con el desempeño de los PCT, se tuvieron en cuenta como variables independientes las siguientes: 
1. Diversidad de los sectores tecnológicos de las empresas ubicadas en el PCT, medida a través del conteo de diferentes sectores tecnológicos a los que pertenecen las firmas que se encuentran en él.

2. El tamaño de la infraestructura del PCT, medida por el área total construida en metros cuadrados.

3. La edad del parque, medida por el número de años transcurridos desde la creación del PCT hasta 2007.

4. La presencia de centros tecnológicos y de investigación y desarrollo, medida por el número de centros de $\mathrm{I}+\mathrm{D}$ presentes en el PCT.

5. El esfuerzo en incubación de empresas, medido a través de dos variables:
a. La planta de personal en la(s) incubadora(s) de empresas presente(s) en el PCT.
b. El número de incubadoras existentes en el PCT.

Como factores externos, considerando variables de control asociadas al país donde se encuentran ubicados los PCT, se tienen:

1. El total nacional de solicitudes de patentes.

2. La población del país.

3. El producto interno bruto real en miles de millones de dólares estadounidenses de 2005.

4. El total de la fuerza laboral.

Al igual que los datos de los factores internos, los valores de las variables de control corresponden a 2007 y fueron extraídos de sistema de consulta de datos del Banco Mundial. Esto con excepción del producto interno bruto real, que se obtuvo del Servicio de Investigación Económica del Departamento de Agricultura de los Estados Unidos. En las tablas 2 y 3 se resumen los aspectos más importantes de las variables del estudio.

Tabla 2. Resumen de los aspectos más importantes de las variables analizadas

\begin{tabular}{l|l|c}
\multicolumn{1}{c|}{ Variable } & \multicolumn{1}{c|}{ Medición } & \multicolumn{1}{c}{ Fuente } \\
\hline \multicolumn{2}{c|}{ 1. Variables dependientes } \\
$\begin{array}{l}\text { Desempeño } \\
\text { financiero }\end{array}$ & Ingresos anuales totales recibidos (US\$) & \\
\hline $\begin{array}{l}\text { Desempeño } \\
\text { innovador }\end{array}$ & Patentes comercializadas (\#) & \multirow{2}{*}{ WAINOVA } \\
\cline { 2 - 2 } $\begin{array}{l}\text { Desempeño en } \\
\text { generación de } \\
\text { empresas }\end{array}$ & Patentes solicitadas (\#) & Empresas graduadas desde la creación del PCT (\#) \\
\cline { 2 - 2 } & Empresas incubadas (\#) & \\
\cline { 2 - 2 } & Empresas residentes (\#) & \\
\hline
\end{tabular}




\begin{tabular}{|c|c|c|}
\hline \multicolumn{3}{|c|}{ 2. Variables independientes } \\
\hline $\begin{array}{l}\text { Diversidad de sec- } \\
\text { tores tecnológicos }\end{array}$ & $\begin{array}{l}\text { Diferentes sectores tecnológicos en los que operan } \\
\text { las empresas instaladas (\#) }\end{array}$ & \multirow{6}{*}{ WAINOVA } \\
\hline $\begin{array}{l}\text { Infraestructura } \\
\text { física }\end{array}$ & Área total construida $\left(\mathrm{m}^{2}\right)$ & \\
\hline Edad del parque & Años transcurridos desde la creación del PCT (\#) & \\
\hline $\begin{array}{l}\text { Presencia de } \\
\text { Centros de } \mathrm{I}+\mathrm{D}\end{array}$ & Centros de I+D presentes en el PCT (\#) & \\
\hline \multirow{2}{*}{$\begin{array}{l}\text { Esfuerzo en } \\
\text { incubación de } \\
\text { empresas }\end{array}$} & Personal dedicado a incubación (\#) & \\
\hline & Incubadoras presentes en el PCT (\#) & \\
\hline \multicolumn{3}{|c|}{ 3. Variables de control } \\
\hline $\begin{array}{l}\text { Solicitudes de } \\
\text { patentes }\end{array}$ & Total nacional de solicitudes (\#) & \multirow[t]{2}{*}{ Banco Mundial } \\
\hline Población del país & Total nacional de habitantes (\#) & \\
\hline PIB real & US\$ 2005 (miles de millones) & $\begin{array}{l}\text { Servicio de Investigación } \\
\text { Económica del Departa- } \\
\text { mento de Agricultura de } \\
\text { Estados Unidos }\end{array}$ \\
\hline $\begin{array}{l}\text { Fuerza laboral } \\
\text { del país }\end{array}$ & Total población en edad de trabajar (\#) & Banco Mundial \\
\hline
\end{tabular}

Fuente: elaboración propia.

Tabla 3. Algunas estadísticas descriptivas de las mediciones en la muestra analizada

\begin{tabular}{l|c|c|c|c|c}
\hline Variables & Recuento & Promedio & $\begin{array}{c}\text { Desviación } \\
\text { estándar }\end{array}$ & Mínimo & Máximo \\
\hline Variables independientes & 378 & 10,7275 & 5,79054 & 1 & 26 \\
\hline Sectores & 334 & $1,12 \mathrm{E}+06$ & $4,97 \mathrm{E}+06$ & 60 & $7,04 \mathrm{E}+07$ \\
\hline Área total construida & 407 & 13,656 & 9,74652 & 1 & 67 \\
\hline Edad & 239 & 9,61506 & 21,3947 & 1 & 200 \\
\hline Centros de I+D & 245 & 822,947 & 9131,02 & 1 & 130000 \\
\hline Personal de incubación & 289 & 3,95156 & 13,421 & 1 & 182 \\
\hline Incubadoras & 83 & $1,11 \mathrm{E}+10$ & $8,52 \mathrm{E}+10$ & 3123,26 & $7,75 \mathrm{E}+11$ \\
\hline Variables dependientes & \multicolumn{7}{|l}{ Continúa } \\
\hline Desempeño financiero
\end{tabular}




Desempeño innovador
\begin{tabular}{l|c|c|c|c|c}
\hline Patentes comercializadas & 57 & 102,86 & 630,598 & 0 & 4766 \\
\hline Patentes solicitadas & 80 & 198,2 & 1409,43 & 1 & 12632 \\
\hline Desempeño en generación de empresas \\
\hline Empresas graduadas & 199 & 40 & 137,831 & 0 & 1347 \\
\hline Empresas incubadas & 256 & 46,2891 & 126,09 & 1 & 1489 \\
\hline Empresas residentes & 397 & 175,542 & 807,96 & 1 & 13629 \\
\hline
\end{tabular}

Fuente: elaboración propia.

\section{Tratamiento de los datos}

Con el soporte de la herramienta informática Statgraphics ${ }^{\circledR}$ Centurion $\mathrm{XV}$, se realizaron los procedimientos de análisis multivariado y modelos lineales generalizados. Por medio de estos procedimientos se establecieron cuatro fases de análisis, que se detallan a continuación, en las que se implementaron correlaciones producto-momento de Pearson, correlaciones por rango de Spearman y veinticuatro modelos de regresión.

En la primera fase, se efectuaron correlaciones de Pearson y de Spearman, y los primeros seis modelos de regresión (uno por cada medición de las variables dependientes) contemplando todas las variables independientes y las de control. La segunda fase fue implementada con seis modelos de regresión contando como variables independientes úni- camente aquellas relacionadas con las hipótesis a contrastar.

En la tercera fase, se analizaron otros seis modelos de regresión teniendo en cuenta las variables cuyas hipótesis están bajo contraste, con excepción de la variable que representa a los centros de $\mathrm{I}+\mathrm{D}$, que fue descartada. Esto se explicará en el apartado de resultados y discusión. En la cuarta fase, se analizaron otros seis modelos de regresión, donde se consideraron como variables independientes, en cada modelo, aquellas que en la fase precedente obtuvieron un valor $\mathrm{p}$ menor o igual a 0,15 .

\section{RESULTADOS Y DISCUSIÓN}

A. Primera fase de análisis

Comprendió tres procedimientos aplicados a todos los datos ya descritos en la metodología: 1) correla- 
ciones producto-momento de Pearson, 2) correlaciones ordinales de Spearman y 3) seis regresiones mediante modelos lineales generalizados (MLG).

En la tabla 4, se pueden observar el coeficiente de correlación (coef), el número de pares de datos utilizados para calcular cada coeficiente (n), y el valor $p$ ( $p$-value), que prueba la significancia estadística de las correlaciones estimadas entre las diversas mediciones acerca del desempeño de los PCT, y las mediciones de las variables consideradas como independientes y de control.

El rango de los coeficientes de correlación producto-momento de Pearson va de $-1 \mathrm{a}+1$, y miden la fuerza de la relación lineal entre las mediciones. El rango de los coeficientes de correlación por rango de Spearman también va de $-1 \mathrm{a}+1$, y miden la fuerza de la asociación entre las variables. A diferencia de las correlaciones de Pearson, los coeficientes de Spearman se calculan a partir del orden de los datos más que de sus propios valores $y$, en consecuencia, son menos sensibles a valores aberrantes que los coeficientes de Pearson.

En la tabla 4 están resaltadas aquellas combinaciones de pares de mediciones que por alguno de los dos métodos empleados tuvieron un va- lor $\mathrm{p}$ menor o igual a 0,01 . Valores $\mathrm{p}$ por debajo de 0,01 indican correlaciones significativamente diferentes de cero, con un nivel de confianza del $99 \%$. Como puede observarse, en lo que respecta a las mediciones de las variables de control, que cuentan con un nivel de confianza del $99 \%$, no se encuentra evidencia significativa que indique la existencia de relación lineal o de asociación del PIB real de un país con alguna de las medidas propuestas para el desempeño de un PCT.

Basados exclusivamente en el coeficiente de correlación de Spearman, con un nivel de confianza del $99 \%$, no se encuentra evidencia significativa de asociación entre las solicitudes de patentes, la población y la fuerza laboral de un país con cinco de las seis medidas propuestas para el desempeño de un PCT (ingresos anuales, patentes comercializadas, patentes solicitadas, empresas graduadas y empresas incubadas).

Porsu parte, la medición de empresas residentes en un PCT se mostró sensible a una correlación negativa de Spearman, con la medición de solicitudes de patentes $(-0,15)$ y la fuerza laboral de un país $(-0,12)$. Dichas correlaciones fueron positivas, con valores de 0,07 y 0,47 , respectivamente, por el método de Pearson, mostrando resultados contradictorios. 
En la literatura revisada no existe ción por medio del análisis de moevidencia que explique relaciones di- delos lineales generalizados, tal y rectas entre estas magnitudes, por lo como se describe a continuación. que se procedió a revisar esta situa-

Tabla 4. Correlaciones de producto-momento de Pearson (P) y correlaciones ordinales de Spearman (S) entre las mediciones de desempeño y las mediciones de las variables independientes y de control

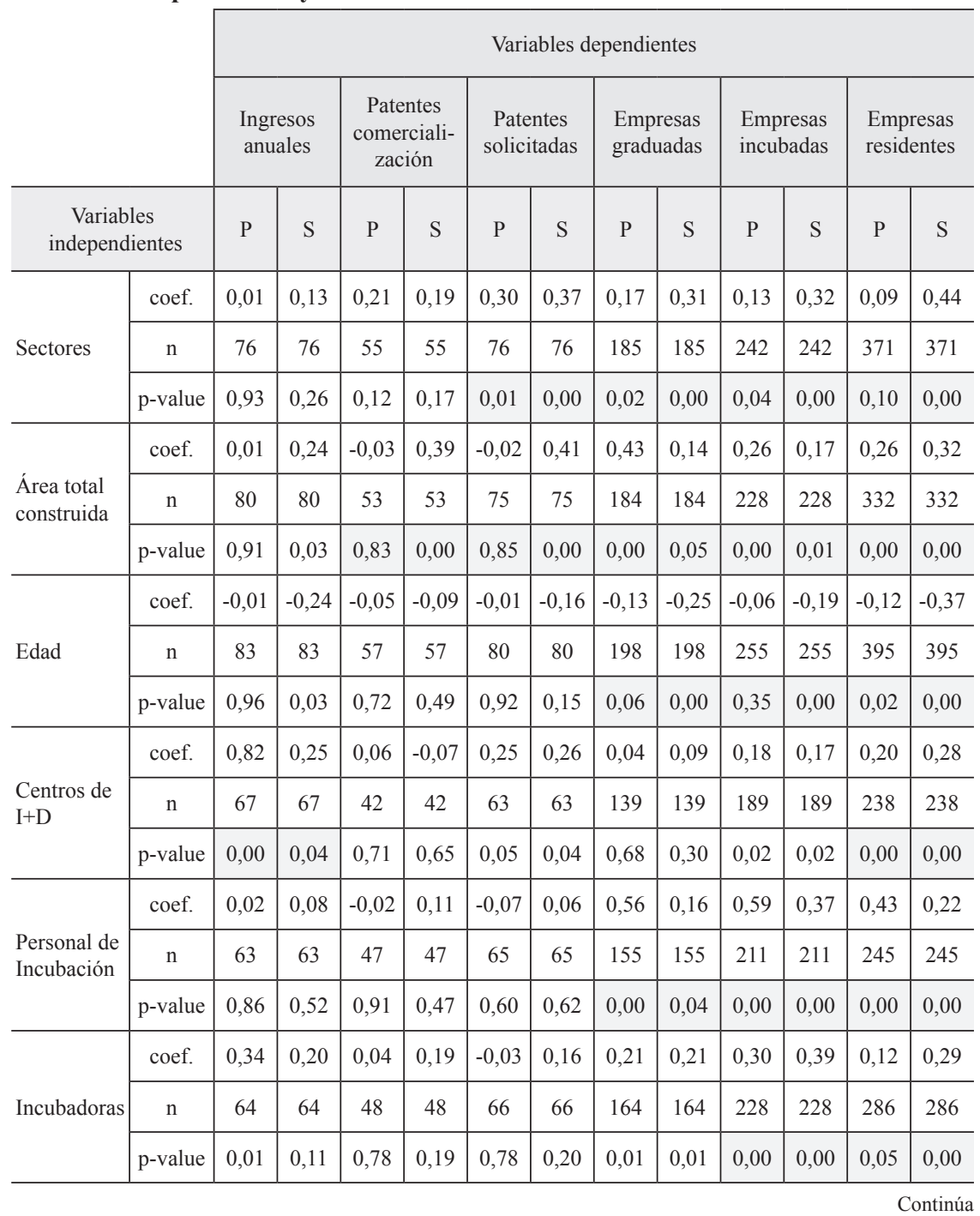




\begin{tabular}{|c|c|c|c|c|c|c|c|c|c|c|c|c|c|}
\hline \multicolumn{2}{|c|}{ Variables de control } & \multirow{2}{*}{$\frac{P}{0,22}$} & \multirow{2}{*}{$\frac{\mathrm{S}}{0,21}$} & \multirow{2}{*}{$\frac{P}{0,46}$} & \multirow{2}{*}{$\frac{S}{0,20}$} & \multirow{2}{*}{$\begin{array}{c}\mathrm{P} \\
0,03\end{array}$} & \multirow{2}{*}{$\frac{S}{0,07}$} & \multirow{2}{*}{$\frac{P}{0,07}$} & \multirow{2}{*}{\begin{tabular}{|c}
$\mathrm{S}$ \\
$-0,17$
\end{tabular}} & \multirow{2}{*}{$\frac{P}{0,13}$} & \multirow{2}{*}{$\frac{\mathrm{S}}{0,04}$} & \multirow{2}{*}{$\frac{P}{0,07}$} & \multirow{2}{*}{$\frac{S}{-0,15}$} \\
\hline \multirow{3}{*}{$\begin{array}{l}\text { Solicitudes } \\
\text { de patentes }\end{array}$} & coef. & & & & & & & & & & & & \\
\hline & $\mathrm{N}$ & 76 & 76 & 51 & 51 & 74 & 74 & 188 & 188 & 242 & 242 & 373 & 373 \\
\hline & $\mathrm{p}$-value & 0,06 & 0,07 & 0,00 & 0,16 & 0,80 & 0,55 & 0,32 & 0,02 & 0,05 & 0,49 & 0,16 & 0,00 \\
\hline \multirow{3}{*}{ Población } & coef. & 0,49 & 0,08 & 0,13 & 0,14 & 0,06 & 0,12 & 0,39 & $-0,08$ & 0,45 & 0,05 & 0,45 & $-0,10$ \\
\hline & $\mathrm{n}$ & 82 & 82 & 56 & 56 & 79 & 79 & 197 & 197 & 254 & 254 & 393 & 393 \\
\hline & $\mathrm{p}$-value & 0,00 & 0,45 & 0,33 & 0,29 & 0,63 & 0,29 & 0,00 & 0,29 & 0,00 & 0,45 & 0,00 & 0,06 \\
\hline \multirow{3}{*}{ PIB real } & coef. & $-0,05$ & 0,12 & $-0,02$ & 0,27 & $-0,05$ & 0,20 & $-0,04$ & $-0,14$ & 0,00 & 0,05 & $-0,03$ & $-0,11$ \\
\hline & $\mathrm{n}$ & 83 & 83 & 57 & 57 & 80 & 80 & 199 & 199 & 256 & 256 & 397 & 397 \\
\hline & p-value & 0,65 & 0,27 & 0,87 & 0,04 & 0,64 & 0,08 & 0,57 & 0,04 & 0,97 & 0,41 & 0,57 & 0,03 \\
\hline \multirow{3}{*}{$\begin{array}{l}\text { Fuerza } \\
\text { laboral }\end{array}$} & coef. & 0,53 & 0,12 & 0,12 & 0,17 & 0,07 & 0,14 & 0,41 & $-0,10$ & 0,48 & 0,07 & 0,47 & $-0,12$ \\
\hline & $\mathrm{n}$ & 82 & 82 & 56 & 56 & 79 & 79 & 197 & 197 & 254 & 254 & 393 & 393 \\
\hline & p-value & 0,00 & 0,28 & 0,36 & 0,19 & 0,56 & 0,22 & 0,00 & 0,18 & 0,00 & 0,25 & 0,00 & 0,01 \\
\hline
\end{tabular}

Fuente: elaboración propia.

Tabla 5: Análisis de Varianza de los seis primeros modelos lineales generalizados: incluyen todas las mediciones (variables independientes y de control).

\begin{tabular}{|c|c|c|c|c|c|c|c|c|c|c|c|c|}
\hline \multirow[b]{3}{*}{ Fuente } & \multicolumn{2}{|c|}{ Modelo A } & \multicolumn{2}{|c|}{ Modelo B } & \multicolumn{2}{|c|}{ Modelo C } & \multicolumn{2}{|c|}{ Modelo D } & \multicolumn{2}{|c|}{ Modelo E } & \multicolumn{2}{|c|}{ Modelo F } \\
\hline & \multicolumn{2}{|c|}{$\begin{array}{l}\mathrm{Ln} \\
\text { (ingresos } \\
\text { anuales) }\end{array}$} & \multicolumn{2}{|c|}{$\begin{array}{c}\text { Ln } \\
\text { (patentes } \\
\text { comercializa- } \\
\text { ción) }\end{array}$} & \multicolumn{2}{|c|}{$\begin{array}{c}\text { Ln } \\
\text { (patentes } \\
\text { solicitadas) }\end{array}$} & \multicolumn{2}{|c|}{$\begin{array}{c}\text { Ln } \\
\text { (empresas } \\
\text { graduadas) }\end{array}$} & \multicolumn{2}{|c|}{$\begin{array}{c}\text { Ln } \\
\text { (empresas } \\
\text { incubadas) }\end{array}$} & \multicolumn{2}{|c|}{$\begin{array}{c}\mathrm{Ln} \\
\text { (empresas } \\
\text { residentes) }\end{array}$} \\
\hline & g.1. & $\mathrm{p}$-value & g.1. & p-value & g.1. & $\mathrm{p}$-value & g.1. & $\mathrm{p}$-value & g.1. & p-value & g.l. & p-value \\
\hline Modelo & 10 & 0,073 & 10 & 0,470 & 10 & 0,010 & 10 & 0,000 & 10 & 0,000 & 10 & 0,000 \\
\hline Sectores & 1 & 0,928 & 1 & 0,164 & 1 & 0,005 & 1 & 0,026 & 1 & 0,000 & 1 & 0,000 \\
\hline $\begin{array}{l}\text { Área total } \\
\text { construida }\end{array}$ & 1 & 0,085 & 1 & 0,863 & 1 & 0,994 & 1 & 0,318 & 1 & 0,195 & 1 & 0,005 \\
\hline Edad & 1 & 0,155 & 1 & 0,555 & 1 & 0,452 & 1 & 0,012 & 1 & 0,045 & 1 & 0,002 \\
\hline $\begin{array}{l}\text { Centros de } \\
\text { I }+D\end{array}$ & 1 & 0,845 & 1 & 0,318 & 1 & 0,482 & 1 & 0,580 & 1 & 0,500 & 1 & 0,263 \\
\hline $\begin{array}{l}\text { Personal de } \\
\text { Incubación }\end{array}$ & 1 & 0,096 & 1 & 0,748 & 1 & 0,463 & 1 & 0,064 & 1 & 0,006 & 1 & 0,005 \\
\hline Incubadoras & 1 & 0,720 & 1 & 0,400 & 1 & 0,597 & 1 & 0,285 & 1 & 0,004 & 1 & 0,194 \\
\hline
\end{tabular}




\begin{tabular}{|c|c|c|c|c|c|c|c|c|c|c|c|c|}
\hline \multirow[b]{3}{*}{$\begin{array}{l}\text { Ln (solicitud } \\
\text { de patente) }\end{array}$} & \multirow{2}{*}{\multicolumn{2}{|c|}{$\begin{array}{c}\text { Modelo A } \\
\begin{array}{c}\text { Ln } \\
\text { (ingresos } \\
\text { anuales) }\end{array}\end{array}$}} & \multirow{2}{*}{\multicolumn{2}{|c|}{$\begin{array}{c}\text { Modelo B } \\
\text { Ln } \\
\text { (patentes } \\
\text { comercializa- } \\
\text { ción) }\end{array}$}} & \multirow{2}{*}{\multicolumn{2}{|c|}{$\begin{array}{c}\text { Modelo C } \\
\begin{array}{c}\text { Ln } \\
\text { (patentes } \\
\text { solicitadas) }\end{array}\end{array}$}} & \multirow{2}{*}{\multicolumn{2}{|c|}{$\begin{array}{c}\text { Modelo D } \\
\text { Ln } \\
\text { (empresas } \\
\text { graduadas) }\end{array}$}} & \multirow{2}{*}{\multicolumn{2}{|c|}{$\begin{array}{c}\text { Modelo E } \\
\text { Ln } \\
\text { (empresas } \\
\text { incubadas) }\end{array}$}} & \multirow{2}{*}{\multicolumn{2}{|c|}{$\begin{array}{c}\text { Modelo F } \\
\text { Ln } \\
\text { (empresas } \\
\text { residentes) }\end{array}$}} \\
\hline & & & & & & & & & & & & \\
\hline & 1 & 0,816 & 1 & 0,948 & 1 & 0,795 & 1 & 0,129 & 1 & 0,549 & 1 & 0,903 \\
\hline Ln (población) & 1 & 0,748 & 1 & 0,588 & 1 & 0,349 & 1 & 0,875 & 1 & 0,357 & 1 & 0,261 \\
\hline Ln (PIB real) & 1 & 0,590 & 1 & 0,179 & 1 & 0,035 & 1 & 0,032 & 1 & 0,109 & 1 & 0,585 \\
\hline $\begin{array}{l}\text { Ln (fuerza } \\
\text { laboral) }\end{array}$ & 1 & 0,767 & 1 & 0,687 & 1 & 0,436 & 1 & 0,920 & 1 & 0,319 & 1 & 0,178 \\
\hline Residuo & 35 & & 14 & & 34 & & 95 & & 132 & & 144 & \\
\hline Total & 45 & & 24 & & 44 & & 105 & & 142 & & 154 & \\
\hline
\end{tabular}

Fuente: elaboración propia.

La tabla 5 muestra los resultados de ajustar6modelosestadísticoslineales generales para relacionar las seis mediciones del desempeño de los PCT, consideradas como variables dependientes con los diez factores predictivos tenidos en cuenta (variables independientes y de control).

Los modelos A y B contemplan a ingresos anuales ( $\mathrm{Ln}$ ) y patentes comercializadas $(\mathrm{Ln})$ como variables dependientes. Teniendo en cuenta que el valor $\mathrm{p}$ para ambos es mayor a 0,01 se obtiene, con un nivel de confianza del $99,0 \%$, que no hay una relación estadísticamente significativa entre estas dos variables dependientes y las diez variables predictoras.

También puede observarse que, en todos los modelos, las mediciones consideradas como controles tienen valores $\mathrm{p}$ mayores que 0,01 . Esto indica que esos términos no son estadísticamente significativos, con un nivel de confianza del 99,0\%. En consecuencia, es plausible eliminarlos del análisis y seguir explorando los restantes como se procedió en la segunda fase.

Adicionalmente, en el tabla 5 puede observarse que los modelos $\mathrm{C}, \mathrm{D}$, $\mathrm{E}$ y $\mathrm{F}$ presentan valores $\mathrm{p}$ menores o iguales a 0,01 , por lo que podría existir una relación estadística significativa. Esto se explora en las fases siguientes.

\section{B. Segunda fase de análisis}

En esta fase se implementaron, nuevamente, seis modelos lineales (uno por cada medición del desempeño) sin considerar los controles propuestos y considerando exclusivamente 
los factores presentes en las hipótesis a contrastar. En la tabla 6 puede observarse que los modelos A1 y B1, correspondientes a ingresos anuales y patentes comercializadas, respectivamente, obtienen valores $p$ por encima de 0,01 . Esto indica una escasa evidencia de relación estadística entre estos indicadores de desempeño de los PCT y las mediciones independientes propuestas en las hipótesis. Estos resultados son consistentes con lo observado en la primera fase.

Estos resultados son también consistentes con la primera fase. Los modelos C1, D1, E1 y F1, los valores $\mathrm{p}$ de estos, son todos menores a 0,01 . Lo anterior indica, con un nivel de confianza del 99,0\%, que existe una relación estadística significativa entre las variables analizadas en cada modelo.

Explorando casos particulares, se desprenden de la tabla 6 los siguientes hallazgos:

1. En el modelo $\mathrm{C} 1$, la diversidad de sectores tecnológicos en un PCT estaría asociada con un mayor desempeño innovador, medido en solicitudes de patentes (valor $\mathrm{p}=0,002$ ).

2. No sorprende que la edad del PCT esté asociada positivamente con un mayor desempeño en materia de emprendimiento. Medido este por medio del número de empresas graduadas desde la creación del parque (valor $\mathrm{p}=$ $0,007)$ en el modelo D1.

3. Tampoco sorprende que un mayor esfuerzo en incubación del PCT (más personal dedicado a incubación y más incubadoras presentes) esté asociado positivamente con un mayor desempeño en materia de emprendimiento. Medido este por medio del número de empresas incubadas (ver modelo E1).

4. También, en el modelo E1, se observa que una mayor diversidad de sectores tecnológicos está asociada positivamente con el número de empresas incubadas.

5. E1 modelo F1 muestra evidencia que los PCT maduros, con mayor diversidad tecnológica y con una infraestructura superior, están asociados igualmente con un mayor desempeño en empresas residentes.

6. No se encontró evidencia de que la cantidad de centros de I+D estuviera asociada con alguna de las medidas de desempeño consideradas de los PCT. Este último hallazgo motivó la realización de una tercera fase de análisis, donde esta no fue tenida en cuenta. 
Tabla 6. Análisis de Varianza de los seis modelos lineales generalizados sin incluir las mediciones de control

\begin{tabular}{|c|c|c|c|c|c|c|c|c|c|c|c|c|}
\hline \multirow[b]{3}{*}{ Fuente } & \multicolumn{2}{|c|}{ Modelo A1 } & \multicolumn{2}{|c|}{ Modelo B1 } & \multicolumn{2}{|c|}{ Modelo C1 } & \multicolumn{2}{|c|}{ Modelo D1 } & \multicolumn{2}{|c|}{ Modelo E1 } & \multicolumn{2}{|c|}{ Modelo F1 } \\
\hline & \multicolumn{2}{|c|}{$\begin{array}{c}\mathrm{Ln} \\
\text { (ingresos } \\
\text { anuales) }\end{array}$} & \multicolumn{2}{|c|}{$\begin{array}{l}\text { Ln } \\
\text { (patentes } \\
\text { comerciali- } \\
\text { zación) }\end{array}$} & \multicolumn{2}{|c|}{$\begin{array}{c}\text { Ln } \\
\text { (patentes } \\
\text { solicitadas) }\end{array}$} & \multicolumn{2}{|c|}{$\begin{array}{c}\text { Ln } \\
\text { (empresas } \\
\text { graduadas) }\end{array}$} & \multicolumn{2}{|c|}{$\begin{array}{c}\text { Ln } \\
\text { (empresas } \\
\text { incubadas) }\end{array}$} & \multicolumn{2}{|c|}{$\begin{array}{c}\text { Ln } \\
\text { (empresas } \\
\text { residentes) }\end{array}$} \\
\hline & g.l. & p-value & g.l. & p-value & g.1. & $\mathrm{p}$-value & g.1. & p-value & g.l. & p-value & g.l. & $\mathrm{p}$-value \\
\hline Modelo & 6 & 0,031 & 6 & 0,588 & 6 & 0,007 & 6 & 0,000 & 6 & 0,000 & 6 & 0,000 \\
\hline Sectores & 1 & 0,808 & 1 & 0,369 & 1 & 0,002 & 1 & 0,073 & 1 & 0,000 & 1 & 0,000 \\
\hline $\begin{array}{l}\text { Área total } \\
\text { construida }\end{array}$ & 1 & 0,023 & 1 & 0,717 & 1 & 0,989 & 1 & 0,550 & 1 & 0,106 & 1 & 0,002 \\
\hline Edad & 1 & 0,042 & 1 & 0,283 & 1 & 0,711 & 1 & 0,007 & 1 & 0,054 & 1 & 0,001 \\
\hline $\begin{array}{l}\text { Centros de } \\
\text { I+D }\end{array}$ & 1 & 0,661 & 1 & 0,230 & 1 & 0,122 & 1 & 0,481 & 1 & 0,605 & 1 & 0,251 \\
\hline $\begin{array}{l}\text { Personal de } \\
\text { incubación }\end{array}$ & 1 & 0,117 & 1 & 0,680 & 1 & 0,366 & 1 & 0,015 & 1 & 0,000 & 1 & 0,019 \\
\hline Incubadoras & 1 & 0,461 & 1 & 0,212 & 1 & 0,321 & 1 & 0,129 & 1 & 0,002 & 1 & 0,144 \\
\hline Residuo & 42 & & 21 & & 42 & & 103 & & 141 & & 155 & \\
\hline Total & 48 & & 27 & & 48 & & 109 & & 147 & & 161 & \\
\hline
\end{tabular}

Fuente: elaboración propia.

\section{Tercera fase de análisis}

La tercera fase de análisis consistió en evaluar la posible asociación entre las mediciones presentes en las hipótesis con excepción de la medición del número de centros de I+D presentes en cada PCT. Lo anterior, al verificarse que no existía evidencia de asociación entre dicha medición con alguna de las mediciones propuestas para el desempeño de parques. La tabla 7 resume los resultados de esta fase de análisis.
Con un nivel de confianza del $99,0 \%$, se pueden considerar los siguientes hallazgos:

1. En concordancia con los resultados de fases de análisis anteriores, en los modelos A2 y B2 no se encuentra evidencia que relacione las mediciones consideradas en las hipótesis con el desempeño financiero de los PCT (medido por ingresos anuales) ni con el desempeño innovador (medido mediante patentes 
comercializadas). En el modelo B2 se encuentra un valor $p$ de 0,006 entre la edad del PCT y las patentes comercializadas por el mismo, lo que indicaría una posible asociación entre ellas. En la cuarta fase se sigue explorando esta relación.

2. También, en concordancia con las anteriores fases, los modelos C2, D2, E2 y F2 muestran valores p muy inferiores a 0,01 , lo que denota significancia estadística en las asociaciones que se dan entre las mediciones de desempeño y las mediciones de las variables presentes en las hipótesis.

3. El modelo $\mathrm{C} 2$ presenta un valor $\mathrm{p}$ de $0,003 \mathrm{y}$, dentro de las fuentes de variación que explican este modelo, aparece que la diversidad de sectores tecnológicos que operan en el PCT es la única medición que presenta una asociación estadísticamente significativa con el desempeño innovador, medido por las patentes solicitadas.

4. El modelo D2 presenta un valor p muy cercano a cero. Esto indicaría suficiente evidencia de asociación entre el desempeño en generación de empresas y las mediciones de diversidad de sectores tecnológicos, la edad del PCT y el personal dedicado a actividades de incubación de empresas.

5. Los resultados del modelo E2 son consistentes con los del modelo E1 de la fase dos, es decir, soportan una asociación estadística significativa entre la diversidad de sectores y el esfuerzo en incubación (en sus dos mediciones) con el desempeño de los PCT, medido por el número de empresas incubadas. En este modelo no se encuentra evidencia de asociación de la edad del parque con el número de empresas incubadas en él.

6. El modelo F2 confirma la asociación estadísticamente significativa del desempeño de los PCT, medido por el número de empresas residentes, con la diversidad de sectores tecnológicos, la infraestructura y la edad del PCT, que se había detectado en el modelo F1 de la fase dos. Además de lo anterior, se detecta la asociación entre el esfuerzo en incubación (personal dedicado a la incubación) y el número de empresas residentes. 
Tabla 7. Análisis de varianza de los seis modelos lineales generalizados, sin incluir las mediciones de control ni a los centros de $I+D$

\begin{tabular}{|c|c|c|c|c|c|c|c|c|c|c|c|c|}
\hline \multirow[b]{3}{*}{ Fuente } & \multicolumn{2}{|c|}{ Modelo A2 } & \multicolumn{2}{|c|}{ Modelo B2 } & \multicolumn{2}{|c|}{ Modelo C2 } & \multicolumn{2}{|c|}{ Modelo D2 } & \multicolumn{2}{|c|}{ Modelo E2 } & \multicolumn{2}{|c|}{ Modelo F2 } \\
\hline & \multicolumn{2}{|c|}{$\begin{array}{l}\mathrm{Ln} \\
\text { (ingresos } \\
\text { anuales) }\end{array}$} & \multicolumn{2}{|c|}{$\begin{array}{c}\text { Ln } \\
\text { (patentes } \\
\text { comercializa- } \\
\text { ción) }\end{array}$} & \multicolumn{2}{|c|}{$\begin{array}{c}\mathrm{Ln} \\
\text { (patentes } \\
\text { solicitadas) }\end{array}$} & \multicolumn{2}{|c|}{$\begin{array}{c}\mathrm{Ln} \\
\text { (empresas } \\
\text { graduadas) }\end{array}$} & \multicolumn{2}{|c|}{$\begin{array}{c}\mathrm{Ln} \\
\text { (empresas } \\
\text { incubadas) }\end{array}$} & \multicolumn{2}{|c|}{$\begin{array}{c}\mathrm{Ln} \\
\text { (empresas } \\
\text { residentes) }\end{array}$} \\
\hline & g.1. & p-value & g.1. & $\mathrm{p}$-value & g.1. & $\mathrm{p}$-value & g.1. & $\mathrm{p}$-value & g.l. & $\mathrm{p}$-value & g.1. & $\mathrm{p}$-value \\
\hline Modelo & 5 & 0,067 & 5 & 0,048 & 5 & 0,003 & 5 & 0,000 & 5 & 0,000 & 5 & 0,000 \\
\hline Sectores & 1 & 0,584 & 1 & 0,314 & 1 & 0,000 & 1 & 0,008 & 1 & 0,000 & 1 & 0,000 \\
\hline $\begin{array}{l}\text { Área total } \\
\text { construida }\end{array}$ & 1 & 0,042 & 1 & 0,795 & 1 & 0,930 & 1 & 0,239 & 1 & 0,187 & 1 & 0,000 \\
\hline Edad & 1 & 0,023 & 1 & 0,006 & 1 & 0,362 & 1 & 0,002 & 1 & 0,126 & 1 & 0,000 \\
\hline $\begin{array}{l}\text { Personal de } \\
\text { incubación }\end{array}$ & 1 & 0,105 & 1 & 0,766 & 1 & 0,403 & 1 & 0,001 & 1 & 0,008 & 1 & 0,010 \\
\hline Incubadoras & 1 & 0,647 & 1 & 0,258 & 1 & 0,354 & 1 & 0,066 & 1 & 0,001 & 1 & 0,195 \\
\hline Residuo & 51 & & 28 & & 51 & & 129 & & 178 & & 206 & \\
\hline Total & 56 & & 33 & & 56 & & 134 & & 183 & & 211 & \\
\hline
\end{tabular}

Fuente: elaboración propia.

D. Cuarta fase de análisis

Con el interés de confirmar la fuerza y significancia de las asociaciones que se habían detectado en las tres primeras fases, se decidió realizar un último análisis de los seis modelos contemplados, teniendo en consideración solo aquellas mediciones que en la fase tres hubieran arrojado valores $\mathrm{p}$ inferiores a 0,15 . En la tabla 8 se resumen los resultados encontrados.

Los hallazgos en esta fase son consistentes con los de las fases anteriores $\mathrm{y}$, con un nivel de confianza del 99,0\%, se tienen los siguientes resultados:

1. No se encuentra evidencia alguna que soporte una asociación estadísticamente significativa entre las mediciones de las variables independientes consideradas en las hipótesis con el desempeño financiero (ingresos anuales, modeloA3), nicon el desempeño innovador (patentes comercializadas, modelo B3).

2. Existe una asociación estadísticamente significativa entre el número de diversos sectores tecnológicosenun PCTy el desempeño 
innovador (patentes solicitadas, modelo C3).

3. El número de empresas graduadas desde la creación de un PCT está asociado con la diversidad de sectores tecnológicos, con la edad del parque, con el personal de incubación y con el número de incubadoras que operan en él (modelo D3).

4. El número actual de empresas incubadas en un PCT está asociado con la diversidad de sectores tec- nológicos, con el personal de incubación y con el número de incubadoras que operan el parque (modelo E3).

5. El número de empresas residentes en un PCT está asociado con la diversidad de sectores tecnológicos, con el área construida, con la edad, con el personal de incubación y con el número de incubadoras que operan el parque (modelo F3).

Tabla 8. Análisis de varianza de los seis modelos lineales generalizados, incluyendo exclusivamente las mediciones con valores $p$ inferiores a 0,15 en la fase 3

\begin{tabular}{|c|c|c|c|c|c|c|c|c|c|c|c|c|}
\hline \multirow[b]{3}{*}{ Fuente } & \multicolumn{2}{|c|}{ Modelo A3 } & \multicolumn{2}{|c|}{ Modelo B3 } & \multicolumn{2}{|c|}{ Modelo C3 } & \multicolumn{2}{|c|}{ Modelo D3 } & \multicolumn{2}{|c|}{ Modelo E3 } & \multicolumn{2}{|c|}{ Modelo F3 } \\
\hline & \multicolumn{2}{|c|}{$\begin{array}{l}\quad \mathrm{Ln} \\
\text { (ingresos } \\
\text { anuales) }\end{array}$} & \multicolumn{2}{|c|}{$\begin{array}{c}\mathrm{Ln} \\
\text { (patentes } \\
\text { comercializa- } \\
\text { ción) }\end{array}$} & \multicolumn{2}{|c|}{$\begin{array}{c}\text { Ln } \\
\text { (patentes } \\
\text { solicitadas) }\end{array}$} & \multicolumn{2}{|c|}{$\begin{array}{c}\text { Ln } \\
\text { (empresas } \\
\text { graduadas) }\end{array}$} & \multicolumn{2}{|c|}{$\begin{array}{c}\text { Ln } \\
\text { (empresas } \\
\text { incubadas) }\end{array}$} & \multicolumn{2}{|c|}{$\begin{array}{c}\text { Ln } \\
\text { (empresas } \\
\text { residentes) }\end{array}$} \\
\hline & g.l. & p-value & g.1. & p-value & g.l. & $\mathrm{p}$-value & g.1. & $\mathrm{p}$-value & g.l. & p-value & g.1. & $\mathrm{p}$-value \\
\hline Modelo & 3 & 0,021 & 1 & 0,042 & 1 & 0,000 & 4 & 0,000 & 4 & 0,000 & 4 & 0,000 \\
\hline Sectores & & & & & 1 & 0,000 & 1 & 0,004 & 1 & 0,000 & 1 & 0,000 \\
\hline $\begin{array}{l}\text { Área total } \\
\text { construida }\end{array}$ & 1 & 0,052 & & & & & & & & & 1 & 0,000 \\
\hline Edad & 1 & 0,027 & 1 & 0,042 & & & 1 & 0,001 & 1 & 0,044 & 1 & 0,000 \\
\hline $\begin{array}{l}\text { Personal de } \\
\text { Incubación }\end{array}$ & 1 & 0,088 & & & & & 1 & 0,002 & 1 & 0,001 & 1 & 0,008 \\
\hline Incubadoras & & & & & & & 1 & 0,000 & 1 & 0,000 & & \\
\hline Residuo & 58 & & 47 & & 74 & & 138 & & 193 & & 210 & \\
\hline Total & 61 & & 48 & & 75 & & 142 & & 197 & & 214 & \\
\hline
\end{tabular}

Fuente: elaboración propia.

\section{CONCLUSIÓN}

El objetivo del presente trabajo fue verificar la existencia de una posi- ble relación del desempeño de los PCT con algunos factores internos característicos de estas organizaciones. También se puso bajo con- 
traste el posible efecto de externalidades sobre el desempeño de los PCT. El desempeño de estos fue analizado desde tres perspectivas: desempeño financiero, desempeño innovador y desempeño en generación de empresas.

Basándose en la revisión de la literatura y en los datos empíricos disponibles, se plantearon cinco hipótesis que exploraban la asociación de igual número de variables con el desempeño de PCT, desde las tres perspectivas ya descritas. Para lograr el objetivo planteado se hizo necesaria la implementación del análisis de correlaciones y veinticuatro ejercicios de regresión mediante modelos lineales generalizados, utilizando datos disponibles de 409 PCT, distribuidos en 48 países.

No se encontró evidencia de que las mediciones de las variables de control asociadas al país en donde se ubica el PCT estuvieran asociadas con alguna de las mediciones del desempeño. Los resultados sugieren que la medición del desempeño de PCT, desde la perspectiva de la generación de empresas, es la más sensible a los factores internos explorados, en especial a la diversidad de sectores tecnológicos y a los esfuerzos en incubación. Esto debería permitir a los directivos de PCT implementar estrategias y acciones re- lacionadas con estas variables, existiendo una alta probabilidad de que tengan una incidencia en el desempeño del PCT, en generación de empresas.

Como principales limitaciones se tienen para esta investigación: 1) no se identifican las relaciones causa efecto, se describen únicamente asociaciones entre variables y 2) si bien el indicador de desempeño de compañías residentes es el que mejor absorbe los factores internos, tiene como límite superior el área total del PCT. Esto impediría evaluar de manera adecuada a los PCT que hayan agotado su área disponible y que ya no pueden crecer más.

Como base para futuras investigaciones, se propone que, a partir de los datos con los que se cuenta ahora, es posible analizar qué sectores tecnológicos están asociados a un mejor desempeño. También puede ser interesante considerar como variable de control la existencia de una estrategia explicita de la administración del PCT, para focalizar esfuerzos en alguna de las variables independientes analizadas en el presente trabajo.

\section{REFERENCIAS}

Asociación de Parques Científicos y Tecnológicos de España (APTE) 
(2003). Los parques cientificos y tecnológicos en el centro del sistema de innovación. Málaga: APTE. Recuperado de http:// www.apte.org/documents/libros/ LOS_PARQUES_CIENTIFICOS_Y TECNOLOGICOS_EN_EL_CENTRO DEL SISTEMA DE INNOVACION.pdf

Association of University Research Parks (AURP) (2011). What is a research park? Recuperado el 11 de octubre de 2012, de http:// www.aurp.net/what-is-a-research-park

Bakouros, Y. L., Mardas, D. C., \& Varsakelis, N. C. (2002). Science park, a high tech fantasy?: an analysis of the science parks of Greece.Technovation,22(2),123128.

Bigliardi, B., Dormio, A., Nosella, A., \& Petroni, G. (2006). Assessing science parks' performances: directions from selected Italian case studies. Technovation, 26(4), 489-505.

Castells, M., \& Hall, P. (1994). Technopoles of the world: The making of 21st century industrial complexes. Nueva York: Routledge.

Chen, C.-J., Wu, H.-L., \& Lin, B.W. (2006). Evaluating the development of high-tech industries: Taiwan's science park. Technological Forecasting and Social Change, 73(4), 452-465.
Colombo,M.,\&Delmastro,M.(2002). How effective are technology incubators? Evidence from Italy. Research Policy, 31(7), 1103 1122.

Dettwiler, P., Lindelöf, P., \& Löfsten, H. (2006). Utility of location: A comparative survey between small new technology-based firms located on and off Science Parks-Implications for facilities management. Technovation, 26(4), 506-517.

Ferguson, R., \&Olofsson, C. (2004). Science parks and the development of NTBFs - Location, survival and growth. The Journal of Technology Transfer, 29(1), 5-17.

Fukugawa, N. (2006). Science parks in Japan and their value-added contributions to new technologybased firms. International Journal of Industrial Organization, 24(2), 381-400.

Hansson, F., Husted, K., \& Vestergaard, J. (2005). Second generation science parks: From structural holes jockeys to social capital catalysts of the knowledge society. Technovation, 25(9), 10391049.

Hu, A. (2007). Technology parks and regional economic growth in China. Research Policy, 36(1), 76-87. International Association of Science Park (IASP) (2002). Knowledge bites. Recuperado el 11 de octu- 
bre de 2012, de http://www.iasp. ws/knowledge-bites

Lindelöf, P., \& Löfsten, H. (2002). Growth, management and financing of new technology-based firms-assessing value-addedcontributions of firms located on and off Science Parks. Omega, 30(3), 143-154.

Lindelöf, P., \& Löfsten, H. (2003). Science park location and new technology-based firms in Sweden - Implications for strategy and performance. SmallBusinessEconomics, 20(3), 245-258.

Lindelöf, P., \& Löfsten, H. (2004). Proximity as a resource base for competitive advantage: University-Industry Links for Technology Transfer. The Journal of Technology Transfer, 29(3/4), 311-326.

Link, A. N., \& Link, K. R. (2003). On the growth of U.S. science parks. The Journal of Technology Transfer, 28(1), 81-85.

Link, A. N., \& Scott, J. T. (2003). The growth of research triangle park. Small Business Economics, 20(2), 167-175.

Löfsten, H., \& Lindelöf, P. (2002). Science parks and the growth of new technology-based firmsacademic-industry links, innovation and markets. Research Policy, 31(6), 859-876.

Löfsten, H., \& Lindelöf, P. (2003). Determinants for an entrepreneurial milieu: Science parks and business policy in growing firms. Technovation, 23(1), 51-64.

Löfsten, H., \& Lindelöf, P. (2005). R\&D networks and product innovation patterns? Academic and non-academic new technologybased firms on science parks. Technovation, 25(9), 1025-1037. Salvador, E. (2011). Are science parks andincubatorsgood"brand names" for spin-offs? The case study of Turin. The Journal of Technology Transfer, 36(2), 203232.

Siegel,D. S., Westhead,P., \&Wright, M. (2003a). Science parks and the performance of new technology-based firms: A review of recent U.K. evidence and an agenda for future research.Small Business Economics, 20(2), 177184.

Siegel, D., Westhead, P., \& Wright, M. (2003b). Assessing the impact of university science parks on research productivity: Exploratory firm-level evidence from the United Kingdom. International Journal of Industrial Organization, 21(9), 1357-1369.

Squicciarini, M. (2009). Science parks: Seedbeds of innovation? A duration analysis of firms' patenting activity. Small Business Economics, 32(2), 169-190.

Storey, D. J., Tether, B. S. (1998). Public policy measures to support new technology-based firms 
in the European Union. Research Policy, 26, 1037-1057.

Sun, C. C. (2011). Evaluating and benchmarking productive performances of six industries in Taiwan Hsin Chu Industrial Science Park. Expert Systems with Applications, 38(3), 2195-2205.

Tan, J. (2006). Growth of industry clusters and innovation: Lessons from Beijing Zhongguancun Science Park. Journal of Business Venturing, 21(6), 827-850.

United Kingdom Science Park Association (UKSPA) (2003). Evaluation of the past and future economic contribution of the UK science park movement. Executive Summary. Essex: UKSPA. Disponible en http://www.tamana.com/apps/cms/articlefiles/70UKSPAEvaluation-ExecutiveSummary.pdf

United Kingdom Science Park Association (UKSPA) (2011). About UKSPA / FAQs about UKSPA.
Recuperado el 11 de octubre de 2012, de http://www.ukspa.org. uk/our-organisation/about-us y http://www.ukspa.org.uk/about ukspa/faqs_about_ukspa/

World Alliance for Innovation (WAINOVA) (2009). WAINOVA Atlas of Innovation: Science/Technology/Research Parks and business incubators in the world. Cheshire: Ten Alps Publishing.

Westhead, P. (1997). R\&D "inputs" and "outputs" of technology-based firms located on and off science parks. $R \& D$ Management, 27 , 45-62.

Westhead,P., Storey,D.J.,(1994).An assessment of firms located on and off science parks in the United Kingdom. Londres: HMSO. Yang, C., Motohashi, K., \& Chen, J. (2009). Are new technologybased firms located on science parks really more innovative? Evidence from Taiwan. Research Policy, 38(1), 77-85. 